\title{
Negative Examples for Sequential Importance Sampling of Binary Contingency Tables
}

\author{
Ivona Bezáková* Alistair Sinclair ${ }^{\dagger} \quad$ Daniel Štefankovič ${ }^{\ddagger} \quad$ Eric Vigoda $^{\S}$
}

June 24, 2011 Keywords: Sequential Monte Carlo; Markov chain Monte Carlo;
Graphs with prescribed degree sequence; Zero-one table

\begin{abstract}
The sequential importance sampling (SIS) algorithm has gained considerable popularity for its empirical success. One of its noted applications is to the binary contingency tables problem, an important problem in statistics, where the goal is to estimate the number of $0 / 1$ matrices with prescribed row and column sums. We give a family of examples in which the SIS procedure, if run for any subexponential number of trials, will underestimate the number of tables by an exponential factor. This result holds for any of the usual design choices in the SIS algorithm, namely the ordering of the columns and rows. These are apparently the first theoretical results on the efficiency of the SIS algorithm for binary contingency tables. Finally, we present experimental evidence that the SIS algorithm is efficient for row and column sums that are regular. Our work is a first step in determining the class of inputs for which SIS is effective.
\end{abstract}

\section{Introduction}

Sequential importance sampling is a widely-used approach for estimating the cardinality of a large set of combinatorial objects. It has been applied in a variety of fields, such as protein folding [14], population genetics [10], and signal processing [12]. Binary contingency tables is an application where the virtues of sequential importance sampling have been especially highlighted; see Chen et al. [7]. This is the subject of this note. Given a set of non-negative row sums $\mathbf{r}=\left(r_{1}, \ldots, r_{m}\right)$ and column sums $\mathbf{c}=\left(c_{1}, \ldots, c_{n}\right)$, let $\Omega=\Omega_{\mathbf{r}, \mathbf{c}}$ denote the set of $m \times n 0 / 1$ tables with row sums $\mathbf{r}$ and column sums c. Let $N=\sum_{i} r_{i}$ denote the number of edges in the corresponding bipartite graphs.

*Department of Computer Science, Rochester Institute of Technology, 102 Lomb Memorial Drive, Rochester, NY 14623. Email: ib@cs.rit.edu.

${ }^{\dagger}$ Computer Science Division, University of California, Berkeley, CA 94720. Email: sinclair@cs.berkeley.edu. Supported by NSF grants CCF-1016896 and CCF-0635153.

${ }^{\ddagger}$ Department of Computer Science, University of Rochester, Rochester, NY 14627. Email: stefanko@cs.rochester.edu. Supported by NSF grant CCF-0910415.

${ }^{\S}$ College of Computing, Georgia Institute of Technology, Atlanta, GA 30332. Email: vigoda@cc.gatech.edu. Supported by NSF grants CCF-0830298 and CCF-0910584. 
Our focus is on algorithms for estimating $|\Omega|$. There are algorithms $[11,3]$ for estimating $|\Omega|$ (and sampling (almost) uniformly at random from $\Omega$ ) which provably run in time polynomial in $n$ and $m$ for any row/column sums. We discuss these algorithms, which use Markov chain Monte Carlo (MCMC) methods, in more detail later in the introduction. In this paper, we study a simpler method known as sequential importance sampling (SIS).

SIS has several purported advantages over the more classical Markov chain Monte Carlo (MCMC) method, such as:

Speed: Chen et al. [7] claim that SIS is faster than MCMC algorithms (their paper shows, by experiment, that for the studied inputs, SIS is superior to the MCMC algorithm of [2]; moreover the authors state that they are not aware of any MCMC-based algorithm that achieves similar results in both accuracy and time as SIS). In fact, Blanchet [5] recently proved that SIS requires $O\left(N^{2}\right)$ time when all of the row and column sums are at most $o\left(N^{1 / 4}\right)$ (see Bayati et al. [1] for a related result for a different algorithm). In contrast, we present a simple example where SIS requires an exponentially large (in $n, m$ ) number of samples to give an approximately correct answer. Note that, as mentioned earlier, a MCMC algorithm was presented in $[11,3]$ which is guaranteed to require at most time polynomial in $n, m$ for every input.

Convergence Diagnostic: One of the difficulties in MCMC algorithms is determining when the Markov chain of interest has reached the stationary distribution, unless we have analytical bounds (as in the case of $[11,3]$ ). SIS seemingly avoids such complications since its output is guaranteed to be an unbiased estimator of $|\Omega|$. Unfortunately, it is unclear how many estimates from SIS are needed before we have a guaranteed close approximation of $|\Omega|$. In our example for which SIS requires exponential time, the estimator appears to converge, but it converges to a quantity that is off from $|\Omega|$ by an exponential factor.

Before formally stating our results, we detail the sequential importance sampling approach for contingency tables, following [7]. The general importance sampling paradigm involves sampling from an 'easy' distribution $\mu$ over $\Omega$ that is, ideally, close to the uniform distribution. At every round, the algorithm outputs a table $\mathbf{T}$ along with $\mu(\mathbf{T})$. Since for any $\mu$ whose support is $\Omega$ one has

$$
E[1 / \mu(\mathbf{T})]=|\Omega|,
$$

the algorithm takes many trials and outputs the average of $1 / \mu(\mathbf{T})$ as an estimate of $|\Omega|$. More precisely, let $\mathbf{T}^{(1)}, \ldots, \mathbf{T}^{(t)}$ denote the outputs from $t$ trials of the SIS algorithm. The final estimate is

$$
X_{t}=\frac{1}{t} \sum_{\ell=1}^{t} \frac{1}{\mu\left(\mathbf{T}^{(\ell)}\right)} .
$$

One typically uses a heuristic to determine how many trials $t$ are needed until the estimator has converged to the desired quantity.

The sequential importance sampling algorithm of Chen et al. [7] constructs the table $\mathbf{T}$ in a column-by-column manner. It is not clear how to order the columns optimally, but 
this will not concern us as our negative results will hold for any ordering of the columns. Suppose the procedure is assigning column $i$ conditional on an existing assignment to columns $1, \ldots, i-1$. For $1 \leq j \leq m$, let $r_{j}^{\prime}$ be equal to $r_{j}$ less the total number of 1 's seen in row $j$ in columns $1, \ldots, i-1$. Thus, $r_{1}^{\prime}, \ldots, r_{m}^{\prime}$ are the residual row sums after taking into account the assignments in the first $i-1$ columns.

The procedure of Chen et al. chooses column $i$ from the following probability distribution. The distribution is the projection onto column $i$ of the uniform distribution over assignments to columns $i, \ldots, n$ where the row sums are $r_{1}^{\prime}, \ldots, r_{m}^{\prime}$ and column $i$ sums to $c_{i}$ (but ignoring the column sums $\left.c_{i+1}, \ldots, c_{n}\right)$. The distribution is easy to describe in closed form. Let $\mathbf{T}_{1, i}, \ldots, \mathbf{T}_{m, i} \in\{0,1\}^{m}$ denote the assignment to column $i$, where $\sum_{j} \mathbf{T}_{j, i}=c_{i}$. Let $n^{\prime}=$ $n-i+1$ be the number of not yet assigned columns. Clearly, $\mathbf{T}_{j, i}$ must be 0 for every $j$ with $r_{j}^{\prime}=0$, and $\mathbf{T}_{j, i}$ must be 1 for every $j$ with $r_{j}^{\prime}=n^{\prime}$. Let $J=\left\{j \in\{1, \ldots, m\} \mid 0<r_{j}^{\prime}<n^{\prime}\right\}$, that is, $J$ is the set of rows whose entries are not forced to 0 or 1 . Then, the probability of the assignment $\mathbf{T}_{j, i}$ for $j \in J$ is proportional to

$$
\prod_{j \in J}\left(\frac{r_{j}^{\prime}}{n^{\prime}-r_{j}^{\prime}}\right)^{\mathbf{T}_{j, i}} .
$$

Sampling from this distribution over assignments for column $i$ can be done efficiently by dynamic programming (see Section 3.1 of [7]).

Remark 1. The described procedure may "get stuck", that is, run into a situation when no valid assignment is possible for the $i$-th column. In such case, $1 / \mu\left(\mathbf{T}^{(\ell)}\right)$ is set to zero in (1) for this trial and the procedure moves to the next trial. Chen et al. also devised a more subtle sampling procedure for the $i$-th column which never gets stuck. We do not describe this interesting modification of the procedure, as the two procedures are equivalent for the input instances which we discuss in this paper. The reason is that for our instances even for the distribution given by (2) SIS never gets stuck.

We now state our negative result. This is a simple family of examples where the SIS algorithm will grossly underestimate $|\Omega|$ unless the number of trials $t$ is exponentially large. Our examples will have the form $\left(1,1, \ldots, 1, d_{r}\right)$ for row sums and $\left(1,1, \ldots, 1, d_{c}\right)$ for column sums, where the number of rows is $m+1$, the number of columns is $n+1$, and we require that $m+d_{r}=n+d_{c}$. An important feature of our examples is that they are "bad" examples regardless of whether the SIS procedure constructs the table column-by-column or row-by-row.

Theorem 2. Let $\beta>0, \gamma \in(0,1)$ be constants satisfying $\beta \neq \gamma$ and consider the input instances $\mathbf{r}=(1,1, \ldots, 1,\lfloor\beta m\rfloor), \mathbf{c}=(1,1, \ldots, 1,\lfloor\gamma m\rfloor)$ with $m+1$ rows. Fix any order of columns (or rows, if sequential importance sampling constructs tables row-by-row) and let $X_{t}$ be the random variable representing the estimate of the SIS procedure after $t$ trials of the algorithm, that is, $X_{t}$ is given by (1). There exist constants $s_{1} \in(0,1)$ and $s_{2}>1$ such that for every sufficiently large $m$ and for any $t \leq s_{2}^{m}$,

$$
\operatorname{Pr}\left(X_{t} \geq \frac{\left|\Omega_{\mathbf{r}, \mathbf{c}}\right|}{s_{2}^{m}}\right) \leq 3 s_{1}^{m} .
$$


We mentioned earlier that there are MCMC algorithms which provably run in time polynomial in $n$ and $m$ for any row/column sums. In particular, Jerrum, Sinclair, and Vigoda [11] presented a polynomial time algorithm for estimating the permanent of a non-negative matrix. For the case of 0/1 matrices, their result corresponds to a randomized algorithm, which for a bipartite graph $G$, estimates the number of perfect matchings of $G$ within a multiplicative factor $(1 \pm \epsilon)$ in time polynomial in $|G|$ and $1 / \epsilon$. The binary contingency tables problem studied in this paper can be reduced to counting perfect matchings via a reduction of Tutte [13]. More recently, Bezáková, Bhatnagar and Vigoda [3] presented a related simulated annealing algorithm that works directly with binary contingency tables to solve the problem studied in this paper for all row/column sums, and has an improved polynomial running time compared with [11]. We note that, in addition to being formally asymptotically faster than any exponential time algorithm, a polynomial time algorithm has additional theoretical significance in that it (and its analysis) implies non-trivial insight into the structure of the problem.

As a side note, we remark that even though SIS grossly underestimates the number of binary contingency tables for our examples with $m+1$ rows with row sums $\left(1,1, \ldots, 1, d_{r}\right)$ and $n+1=m+d_{r}-d_{c}+1$ columns with column sums $\left(1,1, \ldots, 1, d_{c}\right)$, it is possible to compute this number exactly using the formula $\left(\begin{array}{c}m \\ d_{c}\end{array}\right)\left(\begin{array}{c}n \\ d_{r}\end{array}\right)\left(m-d_{c}\right)$ ! $+\left(\begin{array}{c}m \\ d_{c}-1\end{array}\right)\left(\begin{array}{c}n \\ d_{r}-1\end{array}\right)\left(m-d_{c}+1\right)$ !.

Some caveats are in order here. Firstly, the above results imply only that MCMC outperforms SIS asymptotically in the worst case; for many inputs, SIS may well be much more efficient. Secondly, the rigorous worst case upper bounds on the running time of the above MCMC algorithms are still far from practical. Chen et al. [7] showed several examples where SIS outperforms MCMC methods. We present a more systematic experimental study of the performance of SIS, focusing on examples where all the row and column sums are identical as well as on the "bad" examples from Theorem 2. Our experiments suggest that SIS is extremely fast on the balanced examples, while its performance on the bad examples confirms our theoretical analysis. Understanding conditions under which SIS performs well is, perhaps, the most interesting open problem in the area. Specific problems include extending the result of [5] to multiway contingency tables [8] and random graphs with prescribed degrees [6].

We also note that the following simple modification of SIS may lead to better performance. Rather than assigning entries in a column-by-column or row-by-row manner, assign at each step either the row or the column with the largest residual sum. It can easily be verified that this enhanced scheme does produce correct results for the input instances in Theorem 2. However, we provide experimental evidence that there are input instances for which even this enhanced strategy fails. These inputs are similar in flavor to those in Theorem 2, but slightly more complicated.

We begin in Section 2 by presenting a few basic lemmas that are used in the analysis of our negative example. In Section 3 we present our main example where SIS is off by an exponential factor, thus proving Theorem 2. Finally, in Section 4 we summarize some experimental results for SIS that support our theoretical analysis. 


\section{Preliminaries}

We will continue to let $\mu(\mathbf{T})$ denote the probability that a table $\mathbf{T} \in \Omega_{\mathbf{r}, \mathbf{c}}$ is generated by sequential importance sampling algorithm. We let $\pi(\mathbf{T})$ denote the uniform distribution over $\Omega_{\mathbf{r}, \mathbf{c}}$, which is the desired distribution.

Before beginning our main proofs we present two straightforward technical lemmas which are used at the end of the proof of the main theorem. The first lemma claims that if a large set of binary contingency tables gets a very small probability under SIS, then SIS is likely to output an estimate which is not much bigger than the size of the complement of this set, and hence very small. For $S \subset \Omega_{\mathbf{r}, \mathbf{c}}$, let $\bar{S}=\Omega_{\mathbf{r}, \mathbf{c}} \backslash S$ denote its complement.

Lemma 3. Let $p \leq 1 / 2$ and let $S \subseteq \Omega_{r, c}$ be such that $\mu(S) \leq p$. Then for any $a>1$, and any $t$, we have

$$
\operatorname{Pr}\left(X_{t} \leq a|\bar{S}|\right) \geq 1-p t-2 / a .
$$

Proof. The probability that all $t$ SIS trials are not in $S$ is at least

$$
(1-p)^{t} \geq 1-p t
$$

(This well-known inequality valid for $p \geq 0$ follows by induction on $t$.)

Let $\mathbf{T}^{(1)}, \ldots, \mathbf{T}^{(t)}$ be the $t$ tables constructed by SIS. Then, with probability at least $1-p t$, we have $\mathbf{T}^{(\ell)} \in \bar{S}$ for all $\ell, 1 \leq \ell \leq t$. Notice that for a table $\mathbf{T}$ constructed by SIS from $\bar{S}$, we have

$$
\mathbf{E}\left(\frac{1}{\mu(\mathbf{T})} \mid \mathbf{T} \in \bar{S}\right)=\frac{|\bar{S}|}{\mu(\bar{S})}
$$

Let $\mathcal{F}$ denote the event that $\mathbf{T}^{(\ell)} \in \bar{S}$ for all $\ell, 1 \leq \ell \leq t$; hence,

$$
\mathbf{E}\left(X_{t} \mid \mathcal{F}\right)=\frac{|\bar{S}|}{\mu(\bar{S})}
$$

We can use Markov's inequality to estimate the probability that SIS returns an answer which is more than a factor of $a$ worse than the expected value, conditioned on the fact that no SIS trial is from $S$ :

$$
\operatorname{Pr}\left(X_{t}>a|\bar{S}| \mid \mathcal{F}\right) \leq \operatorname{Pr}\left(X_{t}>(a / 2) \frac{|\bar{S}|}{\mu(\bar{S})} \mid \mathcal{F}\right) \leq \frac{2}{a},
$$

where in the first inequality we used $\mu(\bar{S}) \geq 1 / 2$.

Finally, removing the conditioning we get:

$$
\begin{aligned}
\operatorname{Pr}\left(X_{t} \leq a|\bar{S}|\right) & \geq \operatorname{Pr}\left(X_{t} \leq a|\bar{S}| \mid \mathcal{F}\right) \operatorname{Pr}(\mathcal{F}) \\
& \geq\left(1-\frac{2}{a}\right)(1-p t) \\
& \geq 1-p t-\frac{2}{a}
\end{aligned}
$$


The second technical lemma shows that if in a row with large sum (linear in $m$ ) there exists a large number of columns (again linear in $m$ ) for which the SIS probability of placing a 1 at the corresponding position differs significantly from the correct probability, then in any subexponential number of trials the SIS estimator will very likely exponentially underestimate the correct answer.

Let $\mathcal{A}_{i-1}$ denote the set of all assignments of $0 / 1$ to columns $1, \ldots, i-1$ such that the column sums are satisfied and none of the row sums are exceeded. Thus, $\mathbf{A} \in \mathcal{A}_{i-1}$ denotes that $\mathbf{A}$ is a specific assignment of $0 / 1$ to the first $i-1$ columns. Finally, for $\mathbf{A} \in \mathcal{A}_{i-1}$, we use the following notation:

$$
\pi\left(\mathbf{T}_{j, i}=1 \mid \mathbf{T}_{i-1}=\mathbf{A}\right)=\frac{\pi\left(S^{\prime \prime}\right)}{\pi\left(S^{\prime}\right)}
$$

where

$$
S^{\prime}=\left\{\mathbf{T} \in \Omega_{\mathbf{r}, \mathbf{c}}: \text { the first } i-1 \text { columns of } \mathbf{T} \text { are the same as } \mathbf{A}\right\},
$$

and

$$
S^{\prime \prime}=\left\{\mathbf{T} \in S^{\prime}: \mathbf{T}_{j, i}=1\right\}
$$

Similarly, we use

$$
\mu\left(\mathbf{T}_{j, i}=1 \mid \mathbf{T}_{i-1}=\mathbf{A}\right)=\frac{\mu\left(S^{\prime \prime}\right)}{\mu\left(S^{\prime}\right)} .
$$

As we mentioned in Remark 1, SIS will never get stuck for our input instances. For such input instances, (3) is the same as the probability that SIS assigns 1 to $\mathbf{T}_{j, i}$, given that the first $i-1$ columns are filled with $\mathbf{A}$.

Lemma 4. Let $\alpha<\beta$ be positive constants. Consider a class of instances of the binary contingency tables problem, parameterized by $m$, with $m+1$ row sums, the last of which is $\lfloor\beta m\rfloor$. The remaining row sums and column sums can be arbitrary as long as the SIS procedure never gets stuck. Suppose that there exist constants $f<g$ and a set I of cardinality $\lfloor\alpha m\rfloor>0$ such that one of the following statements is true:

(i) for every $i \in I$ and any $\mathbf{A} \in \mathcal{A}_{i-1}$,

$$
\pi\left(\mathbf{T}_{m+1, i}=1 \mid \mathbf{T}_{i-1}=\mathbf{A}\right) \leq f<g \leq \mu\left(\mathbf{T}_{m+1, i}=1 \mid \mathbf{T}_{i-1}=\mathbf{A}\right),
$$

(ii) for every $i \in I$ and any $\mathbf{A} \in \mathcal{A}_{i-1}$,

$$
\mu\left(\mathbf{T}_{m+1, i}=1 \mid \mathbf{T}_{i-1}=\mathbf{A}\right) \leq f<g \leq \pi\left(\mathbf{T}_{m+1, i}=1 \mid \mathbf{T}_{i-1}=\mathbf{A}\right) .
$$

Then there exists a constant $b_{1} \in(0,1)$ such that for any constant $1<b_{2}<1 / b_{1}$ and any sufficiently large $m$, for any $t \leq b_{2}^{m}$,

$$
\operatorname{Pr}\left(X_{t} \geq \frac{\left|\Omega_{\mathbf{r}, \mathbf{c}}\right|}{b_{2}^{m}}\right) \leq 3\left(b_{1} b_{2}\right)^{m} .
$$


Proof. We will analyze case (i); the other case follows from analogous arguments.

Let $U_{1}, \ldots, U_{n}$ be the entries in the last row of a uniformly random contingency table with the prescribed row and column sums. (Note that $U_{1}, \ldots, U_{n}$ are random variables.) Similarly, let $V_{1}, \ldots, V_{n}$ be the entries in the last row of a contingency table with the prescribed row and column sums generated by SIS.

The random variable $U_{i}$ is dependent on $U_{j}$ for $j<i$ and $V_{i}$ is dependent on $V_{j}$ for $j<i$. However, for every $i \in I, U_{i}$ is stochastically dominated by $U_{i}^{\prime}$, where $U_{i}^{\prime}, i \in I$ is a set of independent Bernoulli random variables that take value 1 with probability $f$. Similarly, for every $i \in I, V_{i}$ stochastically dominates $V_{i}^{\prime}$, where $V_{i}^{\prime}, i \in I$ is a set of independent Bernoulli random variables that take value 1 with probability $g$.

Now we may use the Chernoff bound (see, e.g., [9], Theorem 1.1). Let $k=\lfloor\alpha m\rfloor$. Then

$$
\operatorname{Pr}\left(\sum_{i \in I} U_{i}^{\prime}-k f>\frac{g-f}{2} k\right) \leq \exp \left(-(g-f)^{2} k / 2\right)
$$

and

$$
\operatorname{Pr}\left(k g-\sum_{i \in I} V_{i}^{\prime}>\frac{g-f}{2} k\right) \leq \exp \left(-(g-f)^{2} k / 2\right) .
$$

Let $S$ be the set of all tables which have less than $k f+(g-f) k / 2=k g-(g-f) k / 2$ ones in the last row of the columns in $I$. Let $b_{1}:=\exp \left(-(g-f)^{2} \alpha / 4\right) \in(0,1)$. Then $\exp \left(-(g-f)^{2} k / 2\right) \leq b_{1}^{m}$ for $m \geq 1 / \alpha$. Thus, by the first inequality, under the uniform distribution over all binary contingency tables the probability of the set $S$ is at least $1-b_{1}^{m}$. However, by the second inequality, SIS constructs a table from the set $S$ with probability at most $b_{1}^{m}$.

We are ready to use Lemma 3 with $S$ as defined above and $p=b_{1}^{m}$. Since under the uniform distribution the probability of $S$ is at least $1-b_{1}^{m}$, we have that $|S| \geq\left(1-b_{1}^{m}\right)\left|\Omega_{\mathbf{r}, \mathbf{c}}\right|$. Let $b_{2} \in\left(1,1 / b_{1}\right)$ be any constant and consider $t \leq b_{2}^{m}$ SIS trials. Let $a=\left(b_{1} b_{2}\right)^{-m}$. Then, by Lemma 3 , with probability at least $1-p t-2 / a \geq 1-3\left(b_{1} b_{2}\right)^{m}$ the SIS procedure outputs a value which is at most an $a b_{1}^{m}=b_{2}^{-m}$ fraction of $\left|\Omega_{\mathbf{r}, \mathbf{c}}\right|$.

\section{Proof of Main Theorem}

In this section we prove Theorem 2. Before we analyze the input instances from Theorem 2, we first consider the following simpler class of inputs.

\subsection{Row sums $(1,1, \ldots, 1, d)$ and column sums $(1,1, \ldots, 1)$}

The row sums are $(1, \ldots, 1, d)$ and the number of rows is $m+1$. The column sums are $(1, \ldots, 1)$ and the number of columns is $n=m+d$. We assume that sequential importance sampling constructs the tables column-by-column. If SIS constructed the tables row-by-row, starting with the row with sum $d$, then it would in fact output the correct number of tables exactly. However, in the next subsection we will use this simplified case as a tool in our analysis of 
the input instances $\left(1, \ldots, 1, d_{r}\right),\left(1, \ldots, 1, d_{c}\right)$, for which SIS must necessarily fail regardless of whether it works row-by-row or column-by-column, and regardless of the order it chooses.

Lemma 5. Let $\beta>0$, and consider an input of the form $(1, \ldots, 1, d),(1, \ldots, 1)$ with $m+1$ rows where $d=\lfloor\beta m\rfloor$. Then there exists a constants $s>1$, such that for any sufficiently large $m$, for any $t \leq s^{m}$,

$$
\operatorname{Pr}\left(X_{t} \geq \frac{\left|\Omega_{\mathbf{r}, \mathbf{c}}\right|}{s^{m}}\right) \leq 3(1 / 2)^{m} .
$$

The idea for the proof of the lemma is straightforward. By the symmetry of the column sums, for large $m$ and $d$ and $\alpha \in(0,1)$ a uniform random table will have about $\alpha d$ ones in the first $\alpha$ cells of the last row, with high probability. We will show that for some $\alpha \in(0,1)$ and $d=\beta m$, sequential importance sampling is very unlikely to put this many ones in the first $\alpha$ n columns of the last row. Therefore, since with high probability sequential importance sampling will not construct any table from a set that is a large fraction of all legal tables, it will likely drastically underestimate the number of tables.

Before we prove the lemma, let us first compare the column distributions arising from the uniform distribution over all binary contingency tables with the SIS distributions. We refer to the column distributions induced by the uniform distribution over all tables as the true distributions. The true probability of 1 in the first column and last row can be computed as the number of tables with 1 at this position divided by the total number of tables. For the sequence of row and column sums specified in the statement of Lemma 5 , let $Z(m, d)$ denote the total number of tables with these row/column sums. Note, $Z(m, d)=\left(\begin{array}{c}n \\ d\end{array}\right) m !=\left(\begin{array}{c}m+d \\ d\end{array}\right) m !$, since a table is uniquely specified by the positions of ones in the last row and the permutation matrix in the remaining rows and corresponding columns. Therefore,

$$
\pi\left(\mathbf{T}_{m+1,1}=1\right)=\frac{Z(m, d-1)}{Z(m, d)}=\frac{\left(\begin{array}{c}
m+d-1 \\
d-1
\end{array}\right) m !}{\left(\begin{array}{c}
m+d \\
d
\end{array}\right) m !}=\frac{d}{m+d}
$$

On the other hand, by the definition of sequential importance sampling, $\operatorname{Pr}\left(\mathbf{T}_{i, 1}=1\right) \propto$ $r_{i} /\left(n-r_{i}\right)$, where $r_{i}$ is the row sum in the $i$-th row. Therefore,

$$
\mu\left(\mathbf{T}_{m+1,1}=1\right)=\frac{\frac{d}{n-d}}{\frac{d}{n-d}+m \frac{1}{n-1}}=\frac{d(m+d-1)}{d(m+d-1)+m^{2}} .
$$

Observe that if $d \approx \beta m$ for some constant $\beta>0$, then for sufficiently large $m$ we have

$$
\mu\left(\mathbf{T}_{m+1,1}=1\right)>\pi\left(\mathbf{T}_{m+1,1}=1\right) .
$$

As we will see, this will be true for a linear number of columns, which turns out to be enough to prove that in polynomial time sequential importance sampling exponentially underestimates the total number of binary contingency tables with high probability.

Proof of Lemma 5. We will find a constant $\alpha$ such that for every column $i<\alpha m$ we will be able to derive an upper bound on the true probability and a lower bound on the SIS probability of 1 appearing at the $(m+1, i)$ position. 
For a partially filled table with columns $1, \ldots, i-1$ assigned, let $d_{i}$ be the remaining sum in the last row and let $m_{i}$ be the number of other rows with remaining row sum 1 (note that this determines the contents $\mathbf{A} \in \mathcal{A}_{i-1}$ of the first $i-1$ columns, up to permutation). Then the true probability of 1 in the $i$-th column and last row can be bounded as

$$
\pi\left(\mathbf{T}_{m+1, i}=1 \mid \mathbf{T}_{i-1}=\mathbf{A}\right)=\frac{d_{i}}{m_{i}+d_{i}} \leq \frac{d}{m+d-(i-1)}=: f(d, m, i),
$$

while the probability under SIS can be bounded as

$$
\begin{aligned}
\mu\left(\mathbf{T}_{m+1, i}=1 \mid \mathbf{T}_{i-1}=\mathbf{A}\right) & =\frac{d_{i}\left(m_{i}+d_{i}-1\right)}{d_{i}\left(m_{i}+d_{i}-1\right)+m_{i}^{2}} \\
& \geq \frac{(d-(i-1))(m+d-i)}{d(m+d-1)+m^{2}} \\
& =: g(d, m, i) .
\end{aligned}
$$

Observe that for fixed $m, d$, the function $f$ is increasing and the function $g$ is decreasing in $i$, for $i<d$.

Recall that we are considering a family of input instances parameterized by $m$ with $d=$ $\lfloor\beta m\rfloor$, for a fixed $\beta>0$. We will consider $i<\alpha m$ for some $\alpha \in(0, \beta)$. Let

$$
\begin{gathered}
f^{\infty}(\alpha, \beta):=\lim _{m \rightarrow \infty} f(d, m, \alpha m)=\frac{\beta}{1+\beta-\alpha} \\
g^{\infty}(\alpha, \beta):=\lim _{m \rightarrow \infty} g(d, m, \alpha m)=\frac{(\beta-\alpha)(1+\beta-\alpha)}{\beta(1+\beta)+1} \\
\triangle_{\beta}:=g^{\infty}(0, \beta)-f^{\infty}(0, \beta)=\frac{\beta^{2}}{(1+\beta)(\beta(1+\beta)+1)}>0
\end{gathered}
$$

and observe that for fixed $\beta, f^{\infty}$ is increasing in $\alpha$ and $g^{\infty}$ is decreasing in $\alpha$, for $\alpha<\beta$. Let $\alpha, 0<\alpha<\beta$ be such that $g^{\infty}(\alpha, \beta)-f^{\infty}(\alpha, \beta) \geq \triangle_{\beta} / 2$. Such an $\alpha$ exists by continuity (we only need to take a small enough $\alpha$ ).

By the above, for any $\epsilon>0$ and sufficiently large $m$, and for any $i<\alpha m$, the true probability is upper-bounded by $f^{\infty}(\alpha, \beta)+\epsilon$ and the SIS probability is lower-bounded by $g^{\infty}(\alpha, \beta)-\epsilon$. For our purposes it is enough to fix $\epsilon=\triangle_{\beta} / 8$. Now we can use Lemma 4 with $\alpha$ and $\beta$ defined as above, $f=f^{\infty}(\alpha, \beta)+\epsilon$ and $g=g^{\infty}(\alpha, \beta)-\epsilon$ (notice that all these constants depend only on $\beta$ ), and $I=\{1, \ldots,\lfloor\alpha m\rfloor\}$. Let $b_{1} \in(0,1)$ be the constant guaranteed by Lemma 4 and let $b_{2}=1 /\left(2 b_{1}\right)$. This finishes the proof of the lemma with $s=b_{2}$.

Remark 6. Notice that every contingency table with row sums $(1,1, \ldots, 1, d)$ and column sums $(1,1, \ldots, 1)$ is binary. Thus, this instance proves that the column-based SIS procedure for general (non-binary) contingency tables [7] has the same flaw as the binary SIS procedure. We expect that the negative example used for Theorem 2 also extends to general (i. e., nonbinary) contingency tables, but the analysis becomes more cumbersome. 


\subsection{Row sums $\left(1,1, \ldots, 1, d_{r}\right)$ and column sums $\left(1,1, \ldots, d_{c}\right)$}

We will now prove our main result, using ideas from the proof of Lemma 5.

Proof of Theorem 2. Recall that we are working with row sums $\left(1,1, \ldots, 1, d_{r}\right)$, where the number of rows is $m+1$, and column sums $\left(1,1, \ldots, 1, d_{c}\right)$, where the number of columns is $n+1=m+1+d_{r}-d_{c}$. We will eventually fix $d_{r}=\lfloor\beta m\rfloor$ and $d_{c}=\lfloor\gamma m\rfloor$, but to simplify our expressions we work with $d_{r}$ and $d_{c}$ for now.

The theorem claims that the SIS procedure fails for an arbitrary order of columns with high probability. We first analyze the case when the SIS procedure starts with columns of sum 1; we shall address the issue of arbitrary column order later. As before, under the assumption that the first column has sum 1, we compute the probabilities of 1 being in the last row for uniform random tables and for SIS respectively. For the true probability, the total number of tables can be computed as $\left(\begin{array}{c}m \\ d_{c}\end{array}\right)\left(\begin{array}{c}n \\ d_{r}\end{array}\right)\left(m-d_{c}\right)$ ! $+\left(\begin{array}{c}m \\ d_{c}-1\end{array}\right)\left(\begin{array}{c}n \\ d_{r}-1\end{array}\right)\left(m-d_{c}+1\right)$ !, since a table is uniquely determined by the positions of ones in the $d_{c}$ column and $d_{r}$ row and a permutation matrix on the remaining rows and columns. Thus we have

$$
\begin{aligned}
\pi\left(\mathbf{T}_{m+1,1}=1\right) & =\frac{\left(\begin{array}{c}
m \\
d_{c}
\end{array}\right)\left(\begin{array}{c}
n-1 \\
d_{r}-1
\end{array}\right)\left(m-d_{c}\right) !+\left(\begin{array}{c}
m \\
d_{c}-1
\end{array}\right)\left(\begin{array}{c}
n-1 \\
d_{r}-2
\end{array}\right)\left(m-d_{c}+1\right) !}{\left(\begin{array}{c}
m \\
d_{c}
\end{array}\right)\left(\begin{array}{c}
n \\
d_{r}
\end{array}\right)\left(m-d_{c}\right) !+\left(\begin{array}{c}
m \\
d_{c}-1
\end{array}\right)\left(\begin{array}{c}
n \\
d_{r}-1
\end{array}\right)\left(m-d_{c}+1\right) !} \\
& =\frac{d_{r}\left(n-d_{r}+1\right)+d_{c} d_{r}\left(d_{r}-1\right)}{n\left(n-d_{r}+1\right)+n d_{c} d_{r}}=: f_{2}\left(m, d_{r}, d_{c}\right) ; \\
\mu\left(\mathbf{T}_{m+1,1}=1\right) & =\frac{\frac{d_{r}}{n-d_{r}}}{\frac{d_{r}}{n-d_{r}}+m \frac{1}{n-1}}=\frac{d_{r}(n-1)}{d_{r}(n-1)+m\left(n-d_{r}\right)}=: g_{2}\left(m, d_{r}, d_{c}\right) .
\end{aligned}
$$

Let $d_{r}=\lfloor\beta m\rfloor$ and $d_{c}=\lfloor\gamma m\rfloor$ for some constants $\beta>0, \gamma \in(0,1)$ (notice that this choice guarantees that $n \geq d_{r}$ and $m \geq d_{c}$, as required). Then, as $m$ tends to infinity, $f_{2}$ approaches

$$
f_{2}^{\infty}(\beta, \gamma):=\frac{\beta}{1+\beta-\gamma}
$$

and $g_{2}$ approaches

$$
g_{2}^{\infty}(\beta, \gamma):=\frac{\beta(1+\beta-\gamma)}{\beta(1+\beta-\gamma)+1-\gamma}
$$

Notice that $f_{2}^{\infty}(\beta, \gamma)=g_{2}^{\infty}(\beta, \gamma)$ if and only if $\beta=\gamma$. Moreover, $f_{2}^{\infty}(\beta, \gamma)<g_{2}^{\infty}(\beta, \gamma)$ if and only if $\beta>\gamma$. Suppose that $\beta>\gamma$, that is, $f_{2}^{\infty}(\beta, \gamma)<g_{2}^{\infty}(\beta, \gamma)$ (the opposite case follows analogous arguments and uses the second part of Lemma 4). As in the proof of Lemma 5 , we can define $\alpha$ such that if the importance sampling does not choose the column with sum $d_{c}$ in its first $\alpha m$ choices, then in any subexponential number of trials it will exponentially underestimate the total number of tables with high probability. Formally, we derive an upper bound on the true probability of 1 being in the last row of the $i$-th column, and a lower bound on the SIS probability of the same event (both conditioned on the fact that the $d_{c}$ column is not among the first $i-1$ columns assigned). Assume that we already assigned the first $i-1$ columns of the table. Let $d_{r}^{(i)}$ be the current residual sum in the last row (that is, $d_{r}^{(i)}$ is $d_{r}$ 
less the number of ones assigned to the last row of columns $1, \ldots, i-1), m_{i}$ be the remaining number of rows with sum 1 , and $n_{i}$ the remaining number of columns with sum 1 (note that this determines the contents $\mathbf{A} \in \mathcal{A}_{i-1}$ of the first $i-1$ columns, up to permutation). Notice that $n_{i}=n-i+1, m \geq m_{i} \geq m-i+1$, and $d_{r} \geq d_{r}^{(i)} \geq d_{r}-i+1$. Then

$$
\begin{aligned}
\pi\left(\mathbf{T}_{m+1, i}=1 \mid \mathbf{T}_{i-1}=\mathbf{A}\right) & =\frac{d_{r}^{(i)}\left(n_{i}-d_{r}^{(i)}+1\right)+d_{c} d_{r}^{(i)}\left(d_{r}^{(i)}-1\right)}{n_{i}\left(n_{i}-d_{r}^{(i)}+1\right)+n_{i} d_{c} d_{r}^{(i)}} \\
& \leq \frac{d_{r}\left(n-d_{r}+1\right)+d_{c} d_{r}^{2}}{(n-i+1)\left(n-i-d_{r}+2\right)+(n-i+1) d_{c}\left(d_{r}-i+1\right)} \\
& \leq \frac{d_{r}\left(n-d_{r}+1\right)+d_{c} d_{r}^{2}}{(n-i)\left(n-i-d_{r}\right)+(n-i) d_{c}\left(d_{r}-i\right)} \\
& =: f_{3}\left(m, d_{r}, d_{c}, i\right) ; \\
\mu\left(\mathbf{T}_{m+1, i}=1 \mid \mathbf{T}_{i-1}=\mathbf{A}\right) & =\frac{d_{r}^{(i)}\left(n_{i}-1\right)}{d_{r}^{(i)}\left(n_{i}-1\right)+m_{i}\left(n_{i}-d_{r}^{(i)}\right)} \\
& \geq \frac{\left(d_{r}-i\right)(n-i)}{d_{r} n+m\left(n-d_{r}\right)} \\
& =: g_{3}\left(m, d_{r}, d_{c}, i\right) .
\end{aligned}
$$

As before, notice that if we fix $m, d_{r}, d_{c}>0$ satisfying $d_{c}<m$ and $d_{r}<n$, then $f_{3}$ is an increasing function and $g_{3}$ is a decreasing function in $i$, for $i<\min \left\{n-d_{r}, d_{r}\right\}$. Recall that $n-d_{r}=m-d_{c}$.

Let $\alpha$ be a number such that $0<\alpha<\min \{1-\gamma, \beta\}$ (we will further specify how $\alpha$ is chosen shortly - it will be small enough to satisfy equations (9) and (10) below). Suppose that $i \leq \alpha m<\min \left\{m-d_{c}, d_{r}\right\}$. Thus, the upper bound on $f_{3}$ in this range of $i$ is $f_{3}\left(m, d_{r}, d_{c}, \alpha m\right)$ and the lower bound on $g_{3}$ is $g_{3}\left(m, d_{r}, d_{c}, \alpha m\right)$. If $d_{r}=\lfloor\beta m\rfloor$ and $d_{c}=\lfloor\gamma m\rfloor$, then the upper bound on $f_{3}$ converges to

$$
f_{3}^{\infty}(\alpha, \beta, \gamma):=\lim _{m \rightarrow \infty} f_{3}\left(m, d_{r}, d_{c}, \alpha m\right)=\frac{\beta^{2}}{(1+\beta-\gamma-\alpha)(\beta-\alpha)}
$$

and the lower bound on $g_{3}$ converges to

$$
g_{3}^{\infty}(\alpha, \beta, \gamma):=\lim _{m \rightarrow \infty} g_{3}\left(m, d_{r}, d_{c}, \alpha m\right)=\frac{(\beta-\alpha)(1+\beta-\gamma-\alpha)}{\beta(1+\beta-\gamma)+1-\gamma}
$$

Let

$$
\triangle_{\beta, \gamma}:=g_{3}^{\infty}(0, \beta, \gamma)-f_{3}^{\infty}(0, \beta, \gamma)=g_{2}^{\infty}(\beta, \gamma)-f_{2}^{\infty}(\beta, \gamma)>0 .
$$

We set $\alpha>0$ to satisfy

$$
g_{3}^{\infty}(\alpha, \beta, \gamma)-f_{3}^{\infty}(\alpha, \beta, \gamma) \geq \triangle_{\beta, \gamma} / 2
$$

(Small enough $\alpha$ will work, by the continuity of $(7)$ and $(8)$ for $\alpha \in(0, \min \{1-\gamma, \beta\}$ ).

Now we can conclude this part of the proof identically to the last paragraph of the proof of Lemma 5. 
It remains to deal with the case when sequential importance sampling picks the $d_{c}$ column within the first $\lfloor\alpha m\rfloor$ columns. Suppose $d_{c}$ appears as the $k$-th column. In this case we focus on the subtable consisting of the last $n+1-k$ columns with sum $1, m^{\prime}$ rows with sum 1 , and one row with sum $d^{\prime}$, an instance of the form $\left(1,1, \ldots, 1, d^{\prime}\right),(1, \ldots, 1)$. We will use arguments similar to the proof of Lemma 5.

First we express $d^{\prime}$ as a function of $m^{\prime}$. The number of rows with row sum 1 decreased by at least $d_{c}-1=\lfloor\gamma m\rfloor-1 \geq \gamma m-2$, and at most by $(\alpha+\gamma) m$. Hence, $(1-\alpha-\gamma) m \leq m^{\prime} \leq$ $(1-\gamma) m+2$. Similarly, $d_{r}-\alpha m \leq d^{\prime} \leq d_{r}$ where $d_{r}=\lfloor\beta m\rfloor \geq \beta m-1$. Let $\beta^{\prime}$ be such that $d^{\prime}=\beta^{\prime} m^{\prime}$. Thus, $(\beta-\alpha-1 / m) /(1-\gamma+2 / m) \leq \beta^{\prime} \leq \beta /(1-\alpha-\gamma)$.

Now we find $\alpha^{\prime}$ such that for any $i \leq \alpha^{\prime} m^{\prime}$ we will be able to derive an upper bound on the true probability and a lower bound on the SIS probability of 1 appearing at position $\left(m^{\prime}+1, i\right)$ of the $(n+1-k) \times m^{\prime}$ subtable, no matter how the first $k$ columns were assigned.

By the derivation in the proof of Lemma 5 (see expressions (4) and (5)), as $m^{\prime}$ (and thus also $m$ ) tends to infinity, the upper bound on the true probability approaches

$$
\begin{aligned}
f^{\infty}\left(\alpha^{\prime}, \beta^{\prime}\right) & =\lim _{m \rightarrow \infty} \frac{\beta^{\prime}}{1+\beta^{\prime}-\alpha^{\prime}} \\
& \leq \lim _{m \rightarrow \infty} \frac{\frac{\beta}{1-\alpha-\gamma}}{1+\frac{\beta-\alpha-\frac{1}{m}}{1-\gamma+\frac{2}{m}}-\alpha^{\prime}} \\
& =\frac{\frac{\beta}{1-\alpha-\gamma}}{1+\frac{\beta-\alpha}{1-\gamma}-\alpha^{\prime}} \\
& =: f_{4}^{\infty}\left(\alpha, \beta, \gamma, \alpha^{\prime}\right)
\end{aligned}
$$

and the lower bound on the SIS probability approaches

$$
\begin{aligned}
g^{\infty}\left(\alpha^{\prime}, \beta^{\prime}\right) & =\lim _{m \rightarrow \infty} \frac{\left(\beta^{\prime}-\alpha^{\prime}\right)\left(1+\beta^{\prime}-\alpha^{\prime}\right)}{\beta^{\prime}\left(1+\beta^{\prime}\right)+1} \\
& \geq \lim _{m \rightarrow \infty} \frac{\left(\frac{\beta-\alpha-\frac{1}{m}}{1-\gamma+\frac{2}{m}}-\alpha^{\prime}\right)\left(1+\frac{\beta-\alpha-\frac{1}{m}}{1-\gamma+\frac{2}{m}}-\alpha^{\prime}\right)}{\frac{\beta}{1-\alpha-\gamma}\left(1+\frac{\beta}{1-\alpha-\gamma}\right)+1} \\
& =\frac{\left(\frac{\beta-\alpha}{1-\gamma}-\alpha^{\prime}\right)\left(1+\frac{\beta-\alpha}{1-\gamma}-\alpha^{\prime}\right)}{\frac{\beta}{1-\alpha-\gamma}\left(1+\frac{\beta}{1-\alpha-\gamma}\right)+1} \\
& =: g_{4}^{\infty}\left(\alpha, \beta, \gamma, \alpha^{\prime}\right) .
\end{aligned}
$$

Let us evaluate $f_{4}^{\infty}$ and $g_{4}^{\infty}$ for $\alpha=\alpha^{\prime}=0$ :

$$
f_{4}^{\infty}(0, \beta, \gamma, 0)=\frac{\frac{\beta}{1-\gamma}}{1+\frac{\beta}{1-\gamma}}
$$

and

$$
g_{4}^{\infty}(0, \beta, \gamma, 0)=\frac{\frac{\beta}{1-\gamma}\left(1+\frac{\beta}{1-\gamma}\right)}{\frac{\beta}{1-\gamma}\left(1+\frac{\beta}{1-\gamma}\right)+1}
$$


Substituting $x$ for $\beta /(1-\gamma)$, we can see that $f_{4}^{\infty}(0, \beta, \gamma, 0)<g_{4}^{\infty}(0, \beta, \gamma, 0)$ since $\beta /(1-\gamma) \geq 0$. Now let $\triangle_{\beta, \gamma}^{\prime}:=g_{4}^{\infty}(0, \beta, \gamma, 0)-f_{4}^{\infty}(0, \beta, \gamma, 0)>0$. By continuity, for small enough $\alpha, \alpha^{\prime}>0$ we have

$$
g_{4}^{\infty}\left(\alpha, \beta, \gamma, \alpha^{\prime}\right)-f_{4}^{\infty}\left(\alpha, \beta, \gamma, \alpha^{\prime}\right) \geq \triangle_{\beta, \gamma}^{\prime} / 2
$$

Now we proceed in a fashion similar to the last paragraph of the proof of Lemma 5. More precisely, let $\epsilon:=\triangle_{\beta, \gamma}^{\prime} / 8$ and let $f:=f_{4}^{\infty}\left(\alpha, \beta, \gamma, \alpha^{\prime}\right)+\epsilon$ and $g:=g_{4}^{\infty}\left(\alpha, \beta, \gamma, \alpha^{\prime}\right)-\epsilon$ be the upper bound (for sufficiently large $m$ ) on the true probability and the lower bound on the SIS probability of 1 appearing at the position $(m+1, i)$ for $i \in I:=\left\{k+1, \ldots, k+\left\lfloor\alpha^{\prime} m^{\prime}\right\rfloor\right\}$. Therefore Lemma 4 with parameters $\alpha^{\prime}, \beta, I$ of size $|I|=\left\lfloor\alpha^{\prime} m^{\prime}\right\rfloor, f$, and $g$ implies the statement of the theorem.

Finally, if the SIS procedure constructs the tables row-by-row instead of column-by-column, symmetrical arguments hold. This completes the proof of Theorem 2.

\section{Experiments}

We performed several experimental tests which show sequential importance sampling to be a promising approach for certain classes of input instances.

We ran the sequential importance sampling algorithm for binary contingency tables, using the following stopping heuristic. Let $N=n+m$. For some $\epsilon, k>0$ we stopped if the last $k N$ estimates were all within a $(1+\epsilon)$ factor of the current estimate. We set $\epsilon=0.01$ and $k=5$.

Figure 1(a) shows the evolution of the SIS estimate as a function of the number of trials on the input with all row and column sums $r_{i}=c_{j}=5$, and $50 \times 50$ matrices. In our simulations we used the more delicate sampling mentioned in Remark 1, which guarantees that the assignment in every column is valid, i. e., such an assignment can always be extended to a valid table (or, equivalently, that the random variable $X_{t}$ is always strictly positive). Five independent runs are depicted, together with the correct number of tables $\approx 1.038 \times 10^{281}$, which we computed exactly. To make the figure legible, the $y$-axis is scaled by a factor of $10^{280}$ and it only shows the range from 10 to 10.7 . Note that the algorithm appears to converge to the correct estimate, and our stopping heuristic appears to capture this behavior.

In contrast, Figure 1(b) depicts the SIS evolution on the negative example from Theorem 2 with $m=300, \beta=0.6$ and $\gamma=0.8$, i. e., the input is $(1, \ldots, 1,179),(1, \ldots, 1,240)$ on a $301 \times 240$ matrix. In this case the correct number of tables is

$$
\left(\begin{array}{l}
300 \\
240
\end{array}\right)\left(\begin{array}{l}
239 \\
179
\end{array}\right)(300-240) !+\left(\begin{array}{l}
300 \\
239
\end{array}\right)\left(\begin{array}{l}
239 \\
178
\end{array}\right)(300-239) ! \approx 9.684 \times 10^{205} .
$$

We ran the SIS algorithm under three different settings: first, we constructed the tables column-by-column where the columns were ordered from the largest sum, as suggested in the paper by Chen et al. [7] (the red curves correspond to three independent runs with this setting); second, we ordered the columns from the smallest sum (the green curves); and third, we constructed the tables row-by-row where the rows were ordered from the largest sum (the blue curves). The $y$-axis is on a logarithmic scale (base 10) and one unit on the $x$-axis corresponds to 1000 SIS trials. We ran the SIS estimates for twice the number of trials 
determined by our stopping heuristic to indicate that the unfavorable performance of the SIS estimator on this example is not the result of a poor choice of stopping heuristic. Notice that even the best estimator differs from the true value by about a factor of 40 , while the blue curves are off by more than a factor of 1000 .

Figure 2 represents the number of trials required by the SIS procedure (computed by our stopping heuristic) on several examples for $n \times n$ matrices. The four curves correspond to 5 , $10,\lfloor 5 \log n\rfloor$ and $\lfloor n / 2\rfloor$-regular row and column sums. The $x$-axis represents $n$, the number of rows and columns, and the $y$-axis captures the required number of SIS trials. For each $n$ and each of these row and column sums, we took 20 independent runs and we plotted the median number of trials. For comparison, in Figure 3 we plotted the estimated running time for our bad example from Theorem 2 (recall that this is likely the running time needed to converge to a wrong value!) for $n+m$ ranging from 20 to 140 and various settings of $\beta, \gamma$ : $0.1,0.5$ (red), 0.5, 0.5 (blue), 0.2, 0.8 (green), and 0.6,0.8 (black). In this case it is clear that the convergence time is considerably slower compared with the examples in Figure 2.

\subsection{Alternating Rows and Columns}

The bad input instances from Theorem 2 can be efficiently handled by an enhanced SIS approach which considers both rows and columns for updating. More precisely, the enhanced SIS algorithm assigns entries to the row or column with the largest residual sum. We believe there are input instances for which this enhanced SIS algorithm requires exponential time, but proving such a result appears to be technically difficult. We instead give experimental evidence that there are such bad input instances for the enhanced SIS algorithm.

Specifically, we conjecture that for the family of inputs of the form $\mathbf{r}=(1,1, \ldots, 1,\lfloor m / 2\rfloor$, $\lfloor m / 2\rfloor, \ldots,\lfloor m / 2\rfloor)$ and $\mathbf{c}=(1,1, \ldots, 1,\lfloor m / 2\rfloor,\lfloor m / 2\rfloor, \ldots,\lfloor m / 2\rfloor)$, where $m$ denotes the overall number of rows and there are $\lfloor m / 2\rfloor$ rows with sum $\lfloor m / 2\rfloor$ and $\lfloor m / 2\rfloor$ columns with sum $\lfloor m / 2\rfloor$, the enhanced SIS strategy fails to converge quickly to $|\Omega|$. A theoretical analysis of the performance on this family of inputs is difficult because, unlike the simpler instances of Theorem 2, the true row and column distributions are apparently rather hard to estimate in this case. Therefore, we opted to perform experiments that suggest that even the enhanced SIS algorithm is inefficient for this class of inputs.

We now describe these experiments. We did 30 million SIS trials for $m=100$, and repeated this 12 times. The estimates of $|\Omega|$ from these 12 experiments are presented in Figure 4 . In the figure it is clear that after 30 million trials these 12 experiments yield quite different estimates of $|\Omega|$, differing by a factor on the order of $10^{3}$. These results strongly suggest that the enhanced SIS algorithm has failed to converge to an estimate of $|\Omega|$ after 30 million trials. Moreover, we believe that the values produced by the enhanced SIS algorithm after 30 million trials are substantial underestimates of the true value of $|\Omega|$; however, since we know of no feasible method for accurately estimating $|\Omega|$ on these examples, we cannot compare the experimental estimates to the true value of $|\Omega|$. 


\section{References}

[1] M. Bayati, J.-H. Kim, and A. Saberi. A Sequential Algorithm for Generating Random Graphs. Algorithmica, 58(4):860-910, 2010.

[2] J. Besag and P. Clifford. Sequential Monte Carlo p-values. Biometrika, 78(2):301-304, 1991.

[3] I. Bezáková, N. Bhatnagar, and E. Vigoda. Sampling Binary Contingency Tables with a Greedy Start. Random Structures and Algorithms, 30(1-2):168-205, 2007.

[4] I. Bezáková, A. Sinclair, D. Štefankovič, and E. Vigoda. Negative Examples for Sequential Importance Sampling of Binary Contingency Tables. Version available on the arXiv at: http://arxiv.org/abs/math/0606650

[5] J. Blanchet. Efficient Importance Sampling for Binary Contingency Tables. Annals of Applied Probability, 19(3):949-982, 2009.

[6] Joseph Blitzstein and Persi Diaconis. A Sequential Importance Sampling Algorithm for Generating Random Graphs with Prescribed Degrees. Internet Mathematics, 6(4):489$522,2010$.

[7] Y. Chen, P. Diaconis, S. Holmes, and J.S. Liu. Sequential Monte Carlo Methods for Statistical Analysis of Tables. Journal of the American Statistical Association, 100:109$120,2005$.

[8] Y. Chen, I. Dinwoodie, and S. Sullivant. Sequential Importance Sampling For Multiway Tables. The Annals of Statistics, 34(1):523-545, 2006.

[9] D. P. Dubhashi and A. Panconesi, Concentration of measure for the analysis of randomized algorithms. Cambridge University Press, New York, 2009.

[10] M. De Iorio, R. C. Griffiths, R. Lebois, and F. Rousset. Stepwise Mutation Likelihood Computation by Sequential Importance Sampling in Subdivided Population Models. Theoretical Population Biology, 68:41-53, 2005.

[11] M. Jerrum, A. Sinclair and E. Vigoda. A Polynomial-time Approximation Algorithm for the Permanent of a Matrix with Non-negative Entries. Journal of the Association for Computing Machinery, 51(4):671-697, 2004.

[12] J. Miguez, and P. M. Djuric. Blind Equalization by Sequential Importance Sampling. Proceedings of the IEEE International Symposium on Circuits and Systems, 845-848, 2002.

[13] W. T. Tutte A short proof of the factor theorem for finite graphs. Canad. J. Math, 6:347-352, 1954. 
[14] J. L. Zhang, and J. S. Liu. A New Sequential Importance Sampling Method and its Application to the Two-dimensional Hydrophobic-Hydrophilic Model. Journal of Chemical Physics, 117(7):3492-3498, 2002. 


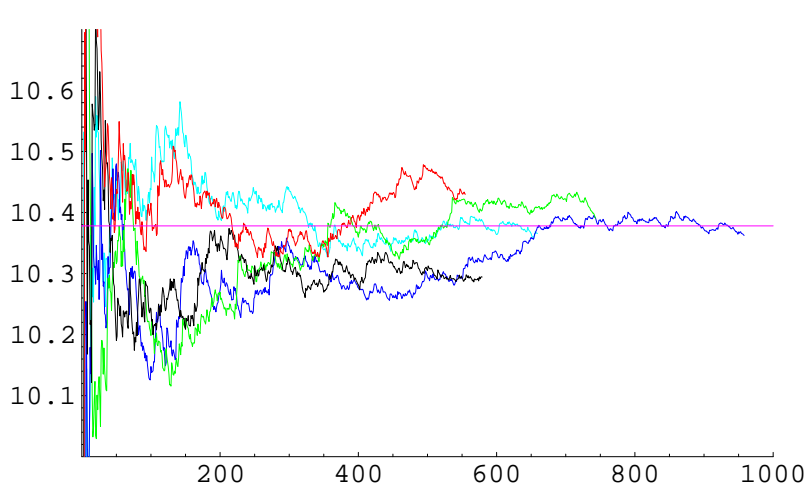

(a)

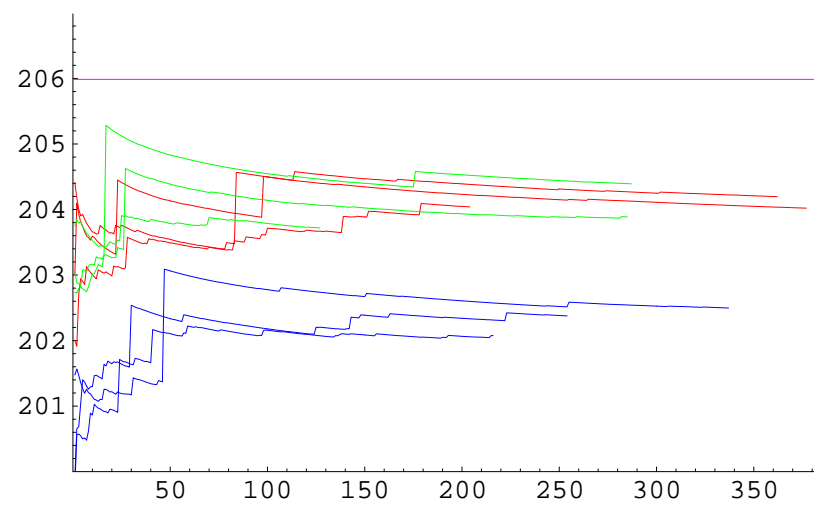

(b)

Figure 1: The estimate produced by sequential importance sampling as a function of the number of trials on two different instances. In both figures, the horizontal line shows the correct number of corresponding binary contingency tables. (a) The left instance is a $50 \times$ 50 matrix where all $r_{i}=c_{j}=5$. The $x$-axis is the number of SIS trials, and the $y$-axis corresponds to the estimate scaled down by a factor of $10^{280}$. Five independent runs of sequential importance sampling are depicted. Notice that the $y$-axis ranges from 10 to 10.7, a relatively small interval, thus it appears SIS converges to the correct estimate. (b) The input instance is from Theorem 2 with $m=300, \beta=0.6$ and $\gamma=0.7$. The estimate ( $y$-axis) is plotted on a logarithmic scale (base 10) and one unit on the $x$-axis corresponds to 1000 SIS trials. Note that in this instance SIS appears to converge to an incorrect estimate. Nine independent runs of the SIS algorithm are shown: the red curves construct tables columnby-column with columns sorted by decreasing sum, the blue curves construct row-by-row with rows sorted by decreasing sum, and the green curves construct column-by-column with columns sorted increasingly.

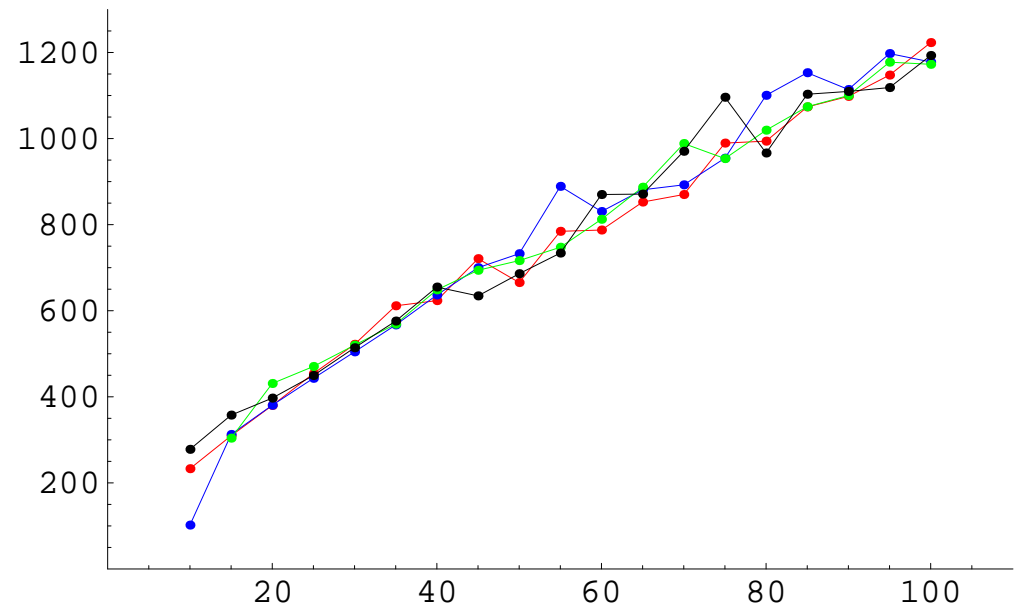

Figure 2: The number of SIS trials before the algorithm converges, as a function of the input size. The curves correspond to 5 (red), 10 (blue), $\lfloor 5 \log n\rfloor$ (green), and $\lfloor n / 2\rfloor$ (black) regular row and column sums. 

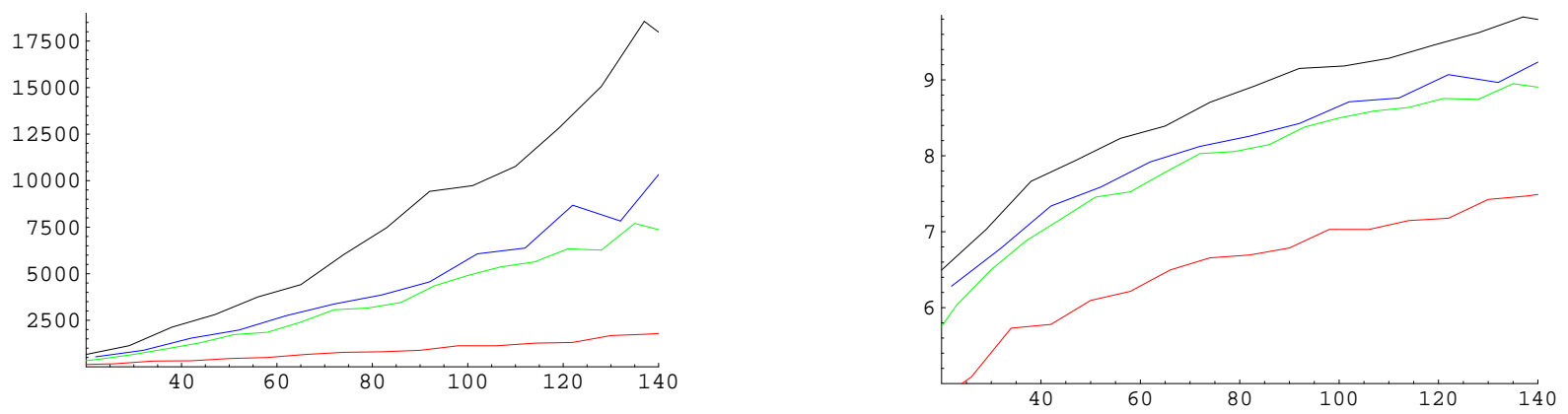

Figure 3: The number of SIS trials until the algorithm converges as a function of $m+n$. The inputs are of the type described in Theorem 2 , with $\beta=0.1, \gamma=0.5$ (red), $\beta=\gamma=0.5$ (blue), $\beta=0.2, \gamma=0.8$ (green), and $\beta=0.6, \gamma=0.8$ (black). The right plot shows the same four curves with the number of SIS trials plotted on a logarithmic scale. Note that the algorithm appears to be converging in sub-exponential time. Recall from Figure 1 that it is converging to the wrong estimate.

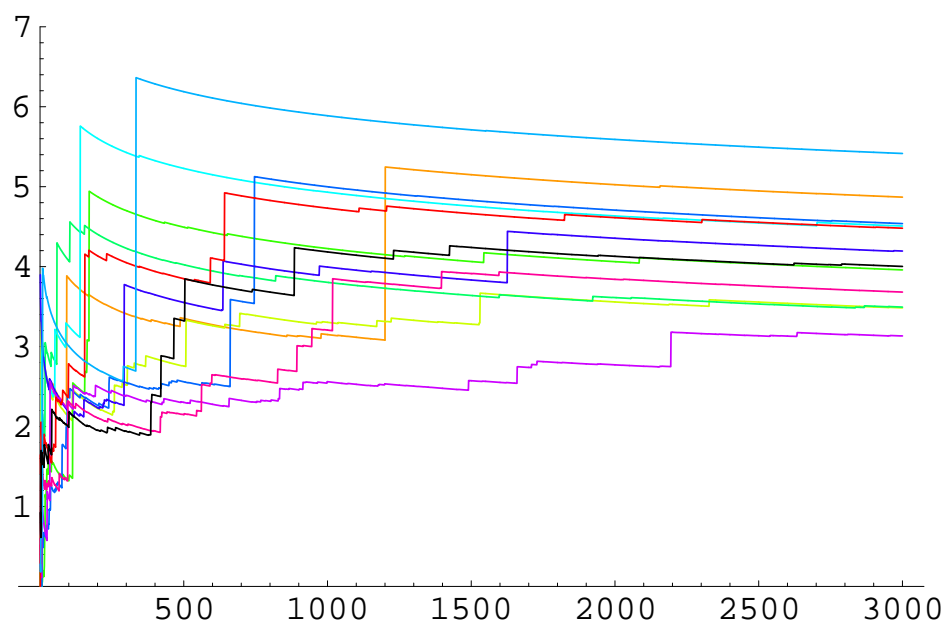

Figure 4: The estimate produced by the enhanced SIS scheme discussed in Section 4.1 as a function of the number of trials for the input $\mathbf{r}=\mathbf{c}=(1,1, \ldots, 1,\lfloor m / 2\rfloor,\lfloor m / 2\rfloor, \ldots,\lfloor m / 2\rfloor)$, where $m$ is the number of rows (and columns), and the number of rows (and columns) with marginal sum $\lfloor m / 2\rfloor$ is $\lfloor m / 2\rfloor$. The $x$-axis depicts the number of SIS trials scaled down by a factor of 10,000, totalling 30,000,000 trials. The $y$-axis depicts the SIS estimate divided by a factor of $10^{200}$, on a logarithmic scale (base 10). Twelve independent runs are shown. 